\title{
Is Bach's brain a Markov chain? Recurrence quantification to assess Markov order for short, symbolic, musical compositions
}

\author{
Jack Murdoch Moore, ${ }^{1}$ Débora Cristina Corrêa, ${ }^{1,2, a)}$ and Michael Small ${ }^{1,2,3}$ \\ ${ }^{1)}$ School of Mathematics and Statistics, The University of Western Australia, Crawley, Western Australia 6009, \\ Australia \\ 2) Complex Data Modelling Group, Faculty of Engineering, Computing and Mathematics, \\ The University of Western Australia, Crawley, Western Australia 6009, Australia \\ 3) Mineral Resources, CSIRO, Kensington, Western Australia 6151, Australia
}

(Dated: 26 June 2018)

It is rarely possible to characterise precisely the system underlying a series of observations. Hypothesis testing, which involves assessing simple assumptions about driving mechanisms, provides hope that we can at least rule out certain possibilities regarding the nature of the system. Unfortunately, the brevity, nonstationarity and symbolic nature of certain time series of interest undermines traditional hypothesis tests. Fortunately, recurrence quantification analysis (RQA) has an established record of success in understanding short and nonstationary time series. We evaluate the suitability of measures of RQA as test statistics in surrogate data tests of the hypothesis that ten compositions by the Baroque composer J. S. Bach (1685 - 1750) arose from a Markov chain. More specifically, we estimate size (the rate at which true hypotheses are incorrectly rejected) and power (the rate at which false hypotheses are correctly rejected) from empirical rejection rates across 1000 realisations, for each of the ten compositions, of the surrogate algorithm. We compare hypothesis tests based on RQA measures to tests based on the conditional entropy, an established test statistic for surrogate data tests of Markov order, and find that the RQA measure $L_{\text {max }}$ provides more consistent rejection of the fairly implausible hypothesis that Bach's brain was a Markov chain.

10 What sort of process underlies music composition? We know that perception of music is mainly controlled by expectation, which in turn is influenced by the presence of recurrent structures. In this study, we investigate the nature of Bach's ${ }_{15}$ compositions by interpreting them as empirical symbolic data arising from a Markov chain; a simple sort of random process which is characterised by its finite memory. Under this hypothesis a random walk on the Markov chain will generate ${ }_{20}$ data with properties similar to those of the original music, which arose from the mind of Bach. Reiteration's important role in music is exploited via recurrence quantification analysis (RQA), a method of data analysis based on measuring pat${ }_{25}$ terns in the recurrence of states. We use RQA to characterise both the original composition and random walks on the Markov chain from which the original composition would arise with greatest likelihood, permitting comparison of the two sorts

30 of time series and thus allowing assessment of the possibility that a composition arose via a random walk. Melodies are short and non-stationary, and thus are time series of a sort with which traditional techniques struggle but with which RQA

35 has a history of success. Our study builds upon this precedent by demonstrating that, compared with an established technique based upon a measure of uncertainty called the conditional entropy,

\footnotetext{
a) Electronic mail: debora.correa@uwa.edu.au
}

RQA provides more reliable rejection of the hy40 pothesis that Bach's remarkable brain was merely a higher order Markov chain.

\section{INTRODUCTION}

Humans may have been appreciating music since before our species existed. ${ }^{1}$ In the modern era, even the 45 melodically-challenged but scientifically-minded can enjoy this ancient art. Diverse disciplines, including neuroscience $^{2}$, psychology ${ }^{3}$, network science ${ }^{4}$ and signal processing ${ }^{5}$, seek a quantitative understanding of music. The complexity of the traditional source of melody, the 50 human mind, motivates application of tools from nonlinear time series analysis, a mathematical discipline which seeks to understand and quantify data from complex sources.

Before unleashing on unknown data the complete 55 panoply of nonlinear time series techniques it would be worthwhile considering the possibility that the data arose in some uncomplicated, and perhaps convenient, manner. A hypothesis test allows assessment of the possibility that a time series of interest arose through a given, 60 relatively simple process. The attraction for scientists of exotic or startling conclusions motivates hypothesis tests for the source of observations. According to Theiler and Prichard ${ }^{6}$, the aim of such hypothesis tests is "to find the least interesting explanation that cannot be invalidated 65 by the data."

A simple hypothesis for symbolic data is that they arose from a discrete Markov chain. A discrete Markov 
chain, which will be defined in section II A, is a random process with a limited memory. The extent of this mem-

70 ory, the number of values present or immediately past which in any way affect the probability distribution of the next value in the sequence, is called the order of the Markov chain.

Markov order is an important modelling parameter ${ }^{7,8}$.

75 In fact, correct estimation of the "memory" of a process is key to successful use of Markov chains in the modelling and forecasting of dynamic process belonging 135 to diverse domains, including computational biology ${ }^{9}$, language processing ${ }^{10}$ and human page navigation mod80 elling $^{11}$. Not surprisingly, Markov order has also been investigated during analysis of temporal dynamics of notes in a melody ${ }^{12-15}$.

Due to its importance, inferring the order of a Markov chain from observed data has received much 85 attention (Please see, for instance, Papapetrou and Kugiumtzis ${ }^{16}$ and their references). Test statistics for discriminating between rival Markov orders follow, in the limit of infinitely many observations, well-understood distributions. ${ }^{17}$ For example, a $\chi^{2}$ distribution describes 90 asymptotically the negative log of the square of the proportional change in maximum likelihood as the assumed Markov order decreases from the true order ${ }^{18}$. In order to avoid applying asymptotic assumptions to small data sets, Van der Heyden et al. ${ }^{19}$ test for Markov order using

95 the method of surrogate data. The method of surrogate data involves generating artificial data which, under the null hypothesis, have properties similar to the original 15 observations which we wish to understand. These artificial data are used to estimate the distribution under 100 the null hypothesis of a chosen test statistic. For their tests of Markov order, Van der Heyden et al. ${ }^{19}$ used as test statistic the conditional entropy, which we define in 160 section II A.

Musical data incur challenges additional to those as105 sociated with other symbolic data. Musical sequences can be short and nonstationary, and additional experimentation cannot augment available data. We cannot ask J. S. Bach to pen for us an extra Sinfonia to decrease the statistical uncertainty in our analysis. Recur110 rence plots (RPs) have a history of success in illuminating data too short or nonstationary for more traditional methods $^{20}$. Recurrence plots have even been used to represent simultaneously the local and global recurrence structure of compositions ${ }^{21}$. For instance, Serra et al. ${ }^{22}$ 115 applied a variant of the RP, called a cross recurrence plot, to identify different versions (covers) of the same song, which suggests that RPs capture something essen- ${ }^{17}$ tial about a composition. These successes make measures which quantify the patterns in RPs attractive discrimi120 nating statistics for hypothesis tests of the source of music data. The application of measures which quantify the patterns in RPs is called recurrence quantification analysis (RQA) and will be introduced in section II C.

Dirst and Weigend ${ }^{23}$ consider the Fugue, a style of ${ }_{125}$ composition for which Bach is well known and which is 180 associated with Markov chains, hypothesis testing and closely related to the styles, the Invention and the Sinfonia, upon which our study focusses ${ }^{24,25}$. They described the structure of Fugues in terms of the arrangement of musical themes (patterns of notes), and transformed ver130 sions of the same themes. Because both the amount of repetition and the amount of novel content in a composition play an important role in music experience $e^{23,26}$, these themes and their images under transformations are combined with non-thematic music material to cre35 ate satisfying harmony and rhythm. Although Dirst and Weigend ${ }^{23}$ did not believe that Fugues emerge from random combinations of the theme and its variations, their explanation of music in terms of "tension between randomness and order" highlights the dual importance of 140 stochasticity and recursion to understanding the process of composition.

Since each set of surrogate data is a realisation of a Markov chain, it is natural to think of them as random walks on weighted networks. McCullough et al. ${ }^{27}$ 45 used RQA measures in investigating the extent to which random walks on a network preserve the character of the time series from which the network arose. Specifically, McCullough et al. ${ }^{27}$ considered random walks on weighted ordinal transition networks constructed from so time series derived from the Lorenz ${ }^{28}$ and other dynamical systems. Campanharo et al. ${ }^{29}$ and Hou et al. ${ }^{30}$ performed analogous investigations of random walks on non-ordinal transition networks, but without employing RQA.

Because the human experience of music depends upon expectation and recurrence ${ }^{31}$, RQA measures have intrinsic significance in descriptions of music. More generally, the relative success of different hypotheses of memory may reflect musical composition conventions.

In this contribution, we benchmark using artificial data the reliability of the method of surrogate data using as test statistics four different RQA measures in addition to the conditional entropy ${ }^{19}$. The results allow assessment of the reliability with which a person analysing a compo5 sition by J. S. Bach would be able to conclude that the source of the time series (the mind of Bach) was not a Markov chain.

The next section introduces briefly concepts, related to Markov chains, the method of surrogate data and recurrence plots, upon which subsequent analysis relies. Section III outlines the approaches used to benchmark hypothesis tests based upon different statistics and to investigate the Markov order of compositions by J. S. Bach. The section which follows presents and interprets 175 the implementation of these approaches. Finally, section $\mathrm{V}$ summarises the main findings and suggests directions for future work.

\section{BACKGROUND}

In this section we introduce briefly important concepts 
recurrence plots.

\section{A. Markov chains}

A random sequence $\left(a_{t}\right)_{t=-\infty}^{\infty}$ indexed by time $t \in \mathbb{Z}$, each element of which takes values in a countable set $S=\left\{A_{i} \mid i \in \mathbb{Z}\right\}$, is called a Markov chain when there exists $n \in \mathbb{N}$ such that the probability distribution of values at each time $t$ depends only upon the value of the variable at the $n$ times which immediately precede time $t$. For fixed $n \in \mathbb{N}$, the necessary condition for a time-homogeneous Markov chain is that

$$
p\left(i_{t} \mid i_{t-1}, i_{t-2}, \ldots\right)=p\left(i_{t} \mid i_{t-1}, \ldots, i_{t-n}\right),
$$

where $p\left(i_{t} \mid i_{t-1}, i_{t-2}, \ldots\right)$ and $p\left(i_{t} \mid i_{t-1}, \ldots, i_{t-n}\right)$ respectively denote the transition probabilities

$$
\mathbb{P}\left(a_{t}=A_{i_{t}} \mid a_{t-1}=A_{i_{t-1}}, a_{t-2}=A_{i_{t-2}}, \ldots\right)
$$

185 and

$$
\mathbb{P}\left(a_{t}=A_{i_{t}} \mid a_{t-1}=A_{i_{t-1}}, \ldots, a_{t-n}=A_{i_{t-n}}\right) .
$$

The order of a Markov chain is the smallest $n$ for which this property holds.

A Markov chain of order $n$ is specified completely by its transition probabilities from words of length $n$

$$
\left\{p\left(i_{t} \mid i_{t-1}, \ldots, i_{t-n}\right) \mid A_{i_{t}}, A_{i_{t-1}}, \ldots, A_{i_{t-n}} \in S\right\} .
$$

${ }_{190}$ That is, these transition probabilities parameterise the space of Markov chains of order $n$ which take values in the set $S$ of symbols.

The quantity which characterises the uncertainty in the value of an element of a Markov chain when the pre195 ceding $m$ values are known is the conditional entropy of order $m$,

$$
\begin{array}{r}
h_{m} \triangleq-\sum_{\left(i_{1}, i_{2}, \ldots, i_{m+1}\right) \in \mathbb{Z}^{m}} p\left(i_{m+1}, i_{m}, \ldots, i_{1}\right) \times \\
\log p\left(i_{m+1} \mid i_{m}, \ldots, i_{1}\right),
\end{array}
$$

where $p\left(i_{m+1}, i_{m}, \ldots, i_{1}\right)$ denotes the probability $\mathbb{P}\left(a_{m+1}=A_{i_{m+1}}, a_{m}=A_{i_{m}}, \ldots, a_{i_{1}}=A_{i_{1}}\right)$. For $\quad$ a Markov chain of order $n$ it follows from (1) that

$$
\text { if } m \geq n \text { then } h_{m}=h_{n} \text {. }
$$

This result is important for hypothesis tests of Markov order which employ as a test statistic the conditional entropy. In particular, in section III C we will employ the preceding inequality. In that section we will also rely upon the following inequality, which applies to all random sequences rather than to Markov chains alone, and which Van der Heyden et al. ${ }^{19}$ attributed to Shannon and Weaver ${ }^{32}$ :

$$
\text { for } m \geq n, \quad h_{m} \leq h_{n}
$$

\section{B. Hypothesis testing and the method of surrogates}

Analysis predicated upon a complicated assumption should be preceded by verification that a simpler assump200 tion is not justified. Hypothesis testing offers a means of assessing simple assumptions.

Testing a null hypothesis $H_{0}$ can involve determining the probability, assuming $H_{0}$, with which a statistic $X$ would be even more extreme than the value $x$ of the 205 statistic which was actually observed. This probability, called the $p$-value, can be calculated from the cumulative distribution function (CDF) of the statistic $X$ under $H_{0}$. If the computed $p$-value is less than or equal to a previously determined probability $\alpha$, called the size of the 210 test, then $H_{0}$ can be rejected at the level of significance $\alpha$. Traditional values of $\alpha$ are $\alpha=0.05$ and $\alpha=0.01$.

The size $\alpha$ of the test is the probability of rejecting $H_{0}$ assuming that this hypothesis is actually correct. This type of mistake is called type $\mathrm{I}$ error $^{33}$ and so size is 215 also known as the rate of type I error. The other sort of mistake which a hypothesis test can incur is type II error $^{33}$; failure to reject $H_{0}$ even though $H_{0}$ is false. The rate of type II error is often written $\beta$ and it is also known as sensitivity. The power of a test is equal to $1-\beta$ and is 220 the probability of rejecting $H_{0}$ when $H_{0}$ is indeed false. The closer to unity is the power the better would the test be able to discriminate against a false null hypothesis. Of course, $\beta$, and hence the power of a test, depends upon the true nature of the observation.

The method of surrogates is a form of hypothesis testing which is particularly useful when the distribution of the statistic $X$ under $H_{0}$ cannot be determined analytically. The method of surrogates uses some number $B$ of instances of the statistic $X$ randomly realised under $H_{0}$, together with the value $x$ originally observed, to estimate the CDF of $X$. This estimate is $F_{X}: \mathbb{R} \rightarrow[0,1]$ given by

$$
F_{X}(y)=\frac{1}{B+1} \sum_{i=0}^{B} \Theta\left(x_{i}-y\right),
$$

${ }_{225}$ where for $i=1,2, \ldots, B, x_{i}$ denotes realisation $i$ of $X$, $x_{0}=x$, and $\Theta$ is the Heaviside step function $\Theta: \mathbb{R} \rightarrow$ $\{0,1\}$ defined by $\Theta(y)=\left\{\begin{array}{ll}0 & \text { if } y<0 \\ 1 & \text { if } y \geq 0\end{array}\right.$.

If only smaller values of a real-valued test statistic $X$ are considered more extreme then the test is called one230 sided and the $p$-value is $p=r /(B+1)$. In this expression $r$ is the rank of $x$, which is the position of $x$ when $x$ and the other $B$ realisations of $X$ are ranked from least to greatest. It follows that the null hypothesis $H_{0}$ can be rejected when the original statistic $x$ lies among the 5 uniquely smallest $\lfloor\alpha(B+1)\rfloor$. If both the smallest and largest values of a real-valued test statistic $X$ are considered extreme then the test is called two-sided and the $p$-value is $p=2 \min \{r /(B+1),(B+2-r) /(B+1)\}$. It follows that the null hypothesis $H_{0}$ can be rejected when 240 the original statistic $x$ lies among either the uniquely largest or the uniquely smallest $\left\lfloor\frac{\alpha}{2}(B+1)\right\rfloor$. 
The requirement for rejection of the null hypothesis that the value of the statistic observed in the original data be strictly more extreme, rather than as or more extreme 245 than all but $\lfloor\alpha(B+1)\rfloor-1$ surrogates, is slightly conservative. If there were ties then, even were the empirical 200 distribution of the test statistic identical with that of the null hypothesis, the true size $\alpha$ could be less than the nominal size $\alpha_{N}$. Van der Heyden et al. ${ }^{19}$ avoided this 250 potential bias by assigning permissible ranks uniformly at random among realisations whose values coincided. Our 205 approach has the advantage that it allows the method of surrogates to be interpreted as proceeding from an empirical estimation via (4) of the cumulative distribution 255 function of the statistic $X$.

\section{Recurrence plots and recurrence quantification analysis}

Consider a series of states $s(1), s(2), \ldots, s(N)$ and a threshold $\epsilon>0$ which corresponds to the distance by which two states must be separated to be distinguishable from one another. When distance $d(s(i), s(j))<\epsilon$ we say that the state at time $i$ recurred at time $j$ or say that the states at $i$ and $j$ are similar. The $N \times N$ matrix $\boldsymbol{R}=\left(R_{i, j}\right)$ defined by

$$
R_{i, j}= \begin{cases}1 & \text { if } d(s(i), s(j)) \leq \epsilon \\ 0 & \text { if } d(s(i), s(j))>\epsilon\end{cases}
$$

is called the similarity or recurrence matrix and captures the patterns of recurrence. These patterns can be visualised using a recurrence plot (RP), which is an ${ }_{260} N \times N$ grid constructed from a recurrence matrix by filling a square at coordinates $(x, y)=(i, j)$ if and only if $R_{i, j}=1$. The line $i=j$ is called the line of identity (LOI). When the distance is a metric, for which always $d(s, s)=0$, the LOI is always filled.

265 If diagonally adjacent coordinates $(i, j)$ and $(i+1, j+1)$ both correspond to recurrent states then it means that the similar states $s(i)$ and $s(j)$ evolved to states $s(i+1)$ and $s(j+1)$ which were also similar. Thus, diagonal lines in a recurrent plot are a hallmark of deterministic evolu270 tion according to a rule which depends continuously on the state. In a periodic system similar states at times $i$ and $j$ can remain similar under forward and reverse time evolution, filling a diagonal line which passes through the coordinate $(i, j)$ and spans the entire RP. In contrast, in 275 deterministic chaos, in which nearby states diverge, diagonal lines on the recurrence plot have limited length. Thus the distribution in a RP of diagonal lines is rich with meaning. Other features of RPs, such as vertical lines, horizontal lines and composite squares, have other 280 interpretations which are outlined, for example, by Marwan et al. ${ }^{34}$.

Eckmann et al. ${ }^{35}$ introduced RPs as a tool for visual characterisation of dynamical systems. Zbilut and Web- 320 ber ${ }^{36}$ provided an alternative to qualitative evaluation ${ }_{285}$ of the features of RPs when they introduced quantitative measures of RP structure. The utilisation and interpretation of these and similar measures is known as recurrence quantification analysis (RQA). For this contribution we consider four RQA measures, which we calculated using the CRP Toolbox for MATLAB ${ }^{\circledR}$. Marwan et al. ${ }^{34}$ provide more information about the toolbox, as well as a more extensive list of RQA measures.

Because of the importance in music of reiteration ${ }^{31}$, we focus upon four RQA measures which quantify the 45 occurrence in a RP of diagonal lines. Hence these RQA measures can be defined in terms of the probability distribution $P: \mathbb{N} \rightarrow[0,1], l \mapsto P(l)$, of lengths $l$ of diagonal lines.

The maximum diagonal line length $L_{\max }$ is the longest 300 observed maintenance of recurrence. Visually, the maximum diagonal line length is the longest diagonal line other than the LOI.

The determinism

$$
D E T=\frac{\sum_{l=l_{\min }}^{\infty} l P(l)}{\sum_{l=1}^{\infty} l P(l)}
$$

is the probability that a randomly chosen pair of similar states will be part of a recurrence of length at least ${ }_{305} l_{\min }$. Visually, the determinism is the fraction of recurrent points which lie on lines of length at least $l_{\min }$.

The mean length of diagonal lines

$$
\langle L\rangle=\frac{\sum_{l=l_{\min }}^{\infty} l P(l)}{\sum_{l=l_{\min }}^{\infty} P(l)}
$$

is the mean length of time for which a new recurrence will be maintained, assuming that it lasts for at least $l_{\min }$ time steps.

In the context of recurrence plots, the entropy usually refers to the Shannon entropy of the distribution of line lengths

$$
E N T R=-\sum_{l=l_{\min }}^{\infty} P(l) \log (P(l)) .
$$

Intuitively, the entropy is the uncertainty in the length of time for which a new recurrence of length at least $l_{\text {min }}$ will be maintained. If recurrences of length at least $l_{\text {min }}$ are distinguished only by the length of time for which they last, then ENTR is the uncertainty in type of a ${ }_{5}$ recurrence which has already been maintained for time $l_{\min }$.

A recurrence plot can also be visualised as a type of complex network called a recurrence network ${ }^{37}$. The nodes of a recurrence network comprise distinct values of the time series, and distinct nodes $s(i)$ and $s(j)$ are linked if and only if $R_{i, j}=1$. That is, nodes $s(i)$ and $s(j)$ are linked if and only if the state at time $i$ recurred 
at time $j$. This interpretation of a RP makes available to RQA the metrics of complex networks ${ }^{37,38}$.

${ }_{325}$ Although in section IV we understand and visualise re- 375 sults using a complex network representation of a musical time series, the directed links of the transition networks ${ }^{37}$ which feature in section IV are defined quite differently from the undirected links of a recurrence network. Our

330 current study does not make use of recurrence network metrics, but they might prove useful test statistics in future work.

\section{METHODS}

Our nominal purpose was to see whether or not Bach's 335 compositions could be distinguished from realisations of Markov chains. More broadly, we were valuating the effectiveness of different discriminating statistics in assessing the nature of short, symbolic time series containing a number of symbols which was relatively large in com340 parison to the number of data. We considered using in the method of surrogate data five different discriminating statistics. These five are the conditional entropy of section II C and the four RQA measures defined in section II A; $L_{\max }, D E T,\langle L\rangle$ and ENTR. Van der Hey345 den et al. ${ }^{19}$ used the method of surrogate data to test hypotheses that short, symbolic time series had Markov order $n$ in the range $0 \leq n \leq 5$. They employed as their test statistic the conditional entropy of order $n$.

Recurrence plots do depend upon the metric with re- ${ }^{400}$ 350 spect to which they are defined, but it was not clear what metric would assign the most meaningful distance between each pair of musical notes. Since the data were discrete, we used the discrete metric defined by $d(A, B)=\left\{\begin{array}{ll}0 & \text { if } A=B \\ 1 & \text { if } A \neq B\end{array}\right.$ and used some fixed threshold

$355 \epsilon \in[0,1)$. A Markov chain of order $n$ produces correlations of length $n$. To focus on correlations not explicitly enforced by the null hypothesis, tests for the hypothesis of Markov order $n$ used $l_{\min }=n+1$. Recurrence quantification analysis with $l_{\min }=2$ on data embedded

360 in dimension $n+1$ produced similar results, as would, likely, many alternative choices of RQA parameters.

\section{A. Selection and preparation of data}

We considered a total of ten keyboard compositions by the composer Johann Sebastian Bach $(1685-1750)^{39}$; 365 five Inventions and five Sinfonias. Bach's Inventions (also known as two-part Inventions) are compositions with two leading voices, while his Sinfonias (also known as three- 420 part Inventions) have three leading voices. Although the different voices are designed to be played simultaneously, 370 each is an independent melody and contains by itself the main characteristics of the composition ${ }^{24,25}$. We derived our time series from the first leading voice of composi- 4 tions. We chose compositions which would lead to symbolic time series of similar length; each Invention led to a time series containing between 228 and 255 symbols, while each Sinfonia led to a time series containing between 221 and 259 symbols. Appendix A provides additional details.

These ten compositions were downloaded in MIDI for380 mat from $I M S L P^{40}$. We used the MIDI Toolbox ${ }^{41}$ to transform these recordings into symbolic time series of discrete notes. In our analysis, each note of a composition comprises a pitch-duration pair. The pitch is the frequency of the sound, while the duration is the length 385 of time between successive notes. A melodic pattern can be repeated at a different tonality, meaning that the pattern recurs translated by some number of units of pitch. In order to recognise recurrence of melodic patterns even when they occurred accross different tonalities, through390 out each composition we transformed the notes such that they were defined with respect to a constant tonality; either the $\mathrm{C}$ major or the A minor tonality. Similarly, we set the beat unit equal to a quarter note to allow recognition of similar rhythmic patterns occurring at different duration ratios. Each pitch-duration pair (each note), distinct modulo tonality, corresponded to a distinct symbol (a distinct node in the network). Our analysis included none of the many other characteristics of music, such as, for example, intensity or timbre ${ }^{42}$.

\section{B. Surrogate algorithm}

The method of surrogate data involves generating new data sets which under the hypothesis $H_{0}$ have properties similar to the data of interest. To this end, when evaluating the null hypothesis that the data $\left(a_{t}\right)_{t=1}^{N}$ arose 405 from a Markov chain of order $n$, we generated $B$ sets of $N$ surrogate data $\left(a_{t}^{(i)}\right)_{t=1}^{N}$ in the following way. To begin with, we appended to the $N$ original data its first $n$ entries, forming the $N+n$ appended data $\left(a_{t}\right)_{t=1}^{N+n}$. The first $n$ entries of the sequence were appended to the ${ }_{410}$ end of the original time series such that $a_{N+t}=a_{t}$ to ensure that no transition probability was left undefined for a word of length $n$ which appeared in the original $N$ data. Next, for each $i=1,2, \ldots, B$ we set $a_{1}^{(i)}=$ $a_{1}, a_{2}^{(i)}=a_{2}, \ldots, a_{n}^{(i)}=a_{n}$ and randomly transitioned to 415 the subsequent $N-n$ symbols $a_{n+1}^{(i)}, a_{n+2}^{(i)}, \ldots, a_{N}^{(i)}$ according to the transition probabilities $\left\{p\left(i_{t} \mid i_{t-1}, \ldots, i_{t-n}\right)\right\}$ with which the appended original data $\left(a_{t}\right)_{t=1}^{N+n}$ would have arisen with maximum likelihood.

The $B$ new data sets arose from a Markov chain of order $n$ so were suitable for testing the hypothesis that the original sequence $\left(a_{t}\right)_{t=1}^{N}$ arose from a Markov chain of this order. However, these surrogate data did not, in general, exhibit transition probabilities from words of length $n$ equal to those of other surrogate data gener5 ated through the same process, or equal to those of the 
original data. We highlight this because in the limit as the number $N$ of data grows large we would expect the observed transition probabilities to approach those of the homogeneous and irreducible ${ }^{43}$ Markov chain from which 430 the data were generated.

The maximum likelihood transition probabilities were straightforward to calculate. As Van der Heyden et al. ${ }^{19}$ explained, for $n \geq 1$ the maximum likelihood transition probabilities are proportional to the observed transitions 435 from each word of length $n$ which appears. When $n=0$ the procedure simply sampled uniformly, with replacement, from the observed density of symbols.

\section{Calculation of rejection rates including size and power}

We rejected the null hypothesis only when the orig440 inal data gave rise to the value of the statistic which ${ }_{405}$ was uniquely most extreme with respect to all statistics which arose from the surrogate and the original data. Neglecting ties, the nominal size $\alpha_{N}$ of such a test is that calculated from the empirical CDF of the statistic. ${ }_{445}$ That is, $\alpha_{N}=\frac{2}{B+1}$ for a two-sided test and $\alpha_{N}=\frac{1}{B+1}$ for a one-sided test. We applied two-sided tests to RQA measures and, following Van der Heyden et al. ${ }^{19}$ in taking advantage of the relationships (2) and (3) of section II A, applied one-sided tests to conditional entropies. To 450 maintain the nominal size $\alpha_{N}$ of the tests, if $B$ sets of ${ }_{505}$ surrogate data were used for RQA measures then only the first $(B-1) / 2$ sets generated were used for the conditional entropy.

There may be differences between the empirical and 455 true CDFs of the test statistic $X$ under a null hypothesis, which can lead to differences between the nominal and true size of the test. We estimated the true size $\alpha$ of the test as the fraction of rejections of the null hypothesis that the process was a Markov process of order $n$ when 460 surrogates were indeed generated from a Markov chain of ${ }_{5}$ order $n$. We estimated the power of the test as the fraction of rejections of the null hypothesis that the process is a Markov process of order $n$ when, in fact, surrogates were generated from a Markov chain of order $n+1$.

465 The ease of tuning the nominal size of a hypothesis 520 test based on surrogate data makes desirable agreement between nominal and true size. Theiler and Prichard ${ }^{44}$ called "accurate" hypothesis tests for which the nominal and true size agree.

470 Rejection rates estimating the size and power were 525 based upon application of the surrogate algorithm to 1000 realisations for each composition. Each realisation was a random realisation of a maximum likelihood Markov chain of the original music data, generated as 475 though by the surrogate algorithm of section III B. In 530 particular, these realisations were of the same length as the original composition.

These estimates of size and power guide interpretation of the rejection rates which were the crux of this project; 480 those calculated from application of the surrogate algo- 5 rithm to each of the ten compositions by J. S. Bach. At each fixed nominal significance $\alpha_{N}$, the hypothesis of each Markov order was assessed only once per composition. Hence, these rejection rates were effectively a 485 simple fraction with a denominator of ten. Those confident that Bach's brain was not a Markov chain may interpret these rejection rates as another estimate of the power of the hypothesis test.

\section{RESULTS AND DISCUSSION}

490 Figures 1 show rates of rejection, using a test with nominal power $\alpha_{N}=0.05$, of the null hypothesis that a time series arose from a Markov process of orders between 0 and 14 . In particular, figures 1 suggest that RQA measures were more suitable than the conditional 5 entropy for testing the hypothesis of Markov order 0. Figures $1 \mathrm{~b}$ and $1 \mathrm{c}$ show that the conditional entropy $h_{0}$, along with all RQA statistics other than DET, led to similar rates of correct rejection of the null hypothesis. However, figure 1a shows that the size $\alpha$ of tests of the 500 hypothesis of a Markov chain of order 0 based upon the conditional entropy $h_{0}$ far exceeded the nominal rejection rate $\alpha_{N}=0.05$. In contrast, tests based upon RQA measures met the criterion $\alpha \leq \alpha_{N}$. Note that when $n=0$, because $L_{\min }=n+1=1$, the determinism $D E T$, defined 5 by (5), was always unity and so could not be expected to lead to rates of rejection other than zero.

For hypotheses of higher Markov order the advantages of RQA measures were present, but were less prominent - figure 1a shows that for order greater than or equal 510 to one the conditional entropy often led to a true size significantly lower than that corresponding to any RQA measure. Figure 1b shows that surrogate data methods based upon RQA measures other than DET exhibited, in benchmarking, power significantly less than that based on conditional entropy. In contrast, for orders one and two DET led to power similar to that corresponding to the conditional entropy, and for orders between three and eight $D E T$ led to a a higher power than did the conditional entropy. Figure 1c details the rates of rejection of hypotheses that original compositions by J. S. Bach arose from Markov processes. This figure shows differences between test statistics very different from those which arose in benchmarking. In practice, at a nominal rate $\alpha_{N}=0.05$, RQA measures allowed, for considerably higher $n$, rejection of the null hypothesis that compositions arose from a Markov chain of order $n$. The conditional entropy allowed rejection of the hypotheses of the compositions having Markov order of one or less at a rate of $100 \%$, but of Markov orders two or higher at rates of $10 \%$ or less. In contrast, three RQA measures - $\langle L\rangle, E N T R$ and, except at order zero, DET - allowed rejection of hypotheses of Markov order four or less at a rate of at least $50 \%$.

Although for fixed nominal size $\alpha_{N}=0.05$ figure 1c 5 shows that the conditional entropy allows for original 


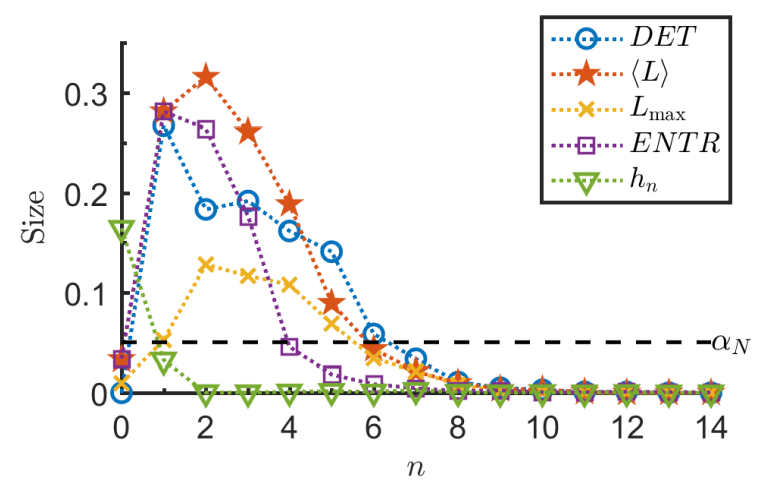

(a)

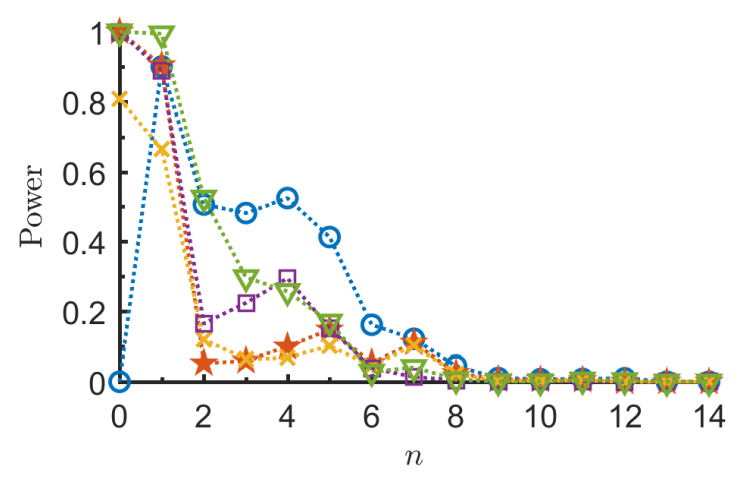

(b)

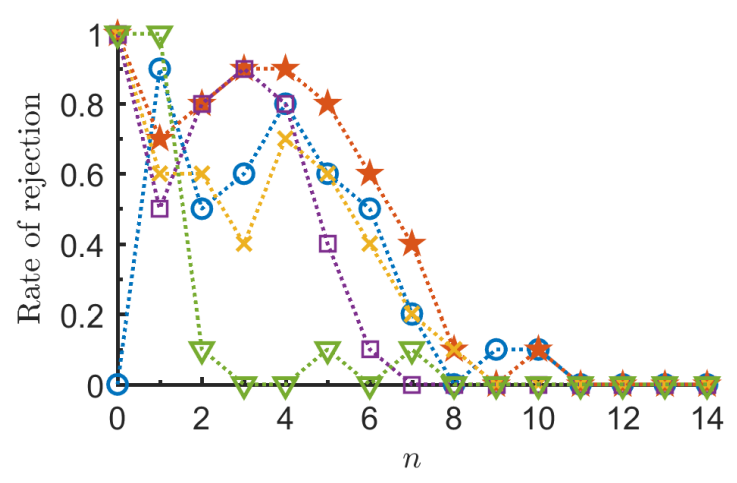

(c)

FIG. 1. Rates of rejection, at a nominal size $\alpha_{N}=0.05$, of the null hypothesis that time series arose from a Markov 550 chain of order $n$ for (a) realisations of 1000 Markov chains of order $n$; (b) realisations of 1000 Markov chains of order $n+1$; and (c) ten compositions by J. S. Bach. For hypotheses involving order $n$ of one or greater, hypothesis tests based on RQA measures exhibit greater size. For order $n$ of two or greater, hypothesis tests based on RQA measures also have better ability to reject the hypothesis that the compositions of Bach arose from a Markov chain of order $n$.

compositions less consistent rejection than do RQA mea- 560 sures of the hypothesis of Markov orders 2-6, figure 1a suggests that it does so with significantly less risk of false rejection; with a lower true size $\alpha$. Comparison of figures ${ }_{540} 2$ and 3 reveals the advantage of RQA measures, and

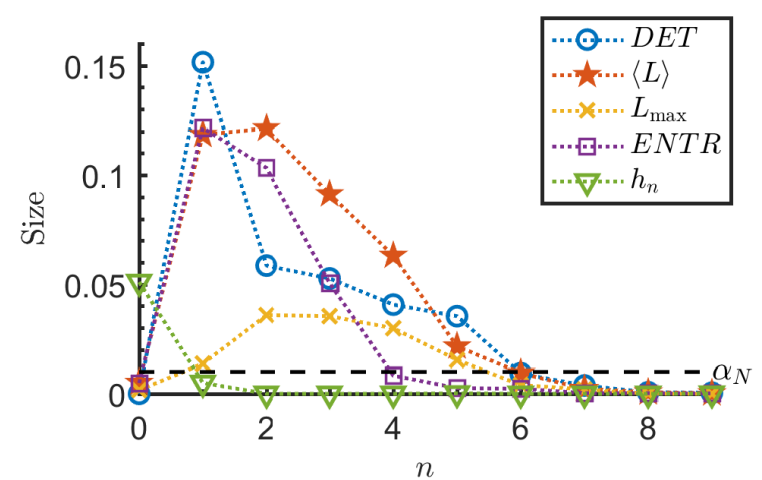

(a)

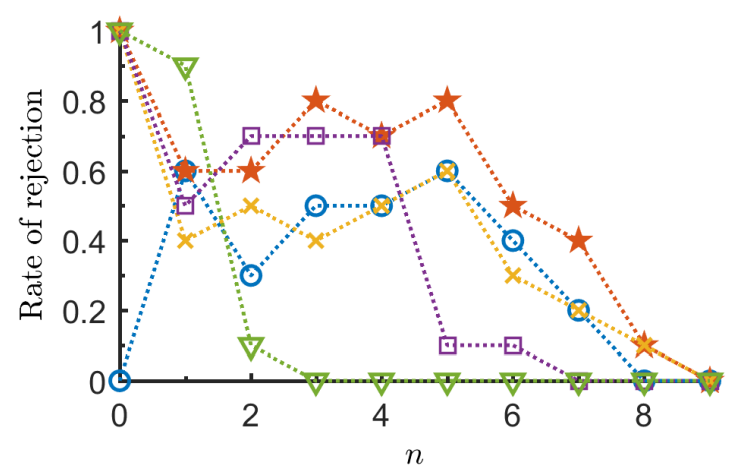

(b)

FIG. 2. Rates of rejection, at a nominal size $\alpha_{N}=0.01$, of the null hypothesis that time series arose from a Markov chain of order $n$ for (a) realisations of 1000 Markov chains of order $n$; and (b) ten compositions by J. S. Bach.

especially the advantage of $L_{\max }$. Figures 2 show that a nominal size $\alpha_{N}=0.01$ provided to hypothesis tests based upon $L_{\max }$ an estimated true size $\alpha$ less than 0.05 alongside a rate of rejection of at least $40 \%$ of null hy${ }_{545}$ potheses of Markov order 3-5. Figures 3 show that, in comparison, a nominal size $\alpha_{N}=0.25$ led, for null hypotheses of some Markov orders in the range 3-5, to tests based upon the conditional entropy with an estimated true size $\alpha$ greater than 0.05 alongside a rate of rejection no more than $20 \%$.

This advantage of $L_{\max }$ in distinguishing musical compositions from random time series is not particularly surprising. The repetition of patterns, with or without variation, is integral to many forms of music, and is especially evident in Bach's Inventions and Sinfonias. This property places RQA in general, and the measure $L_{\max }$ in particular, in a good position to distinguish the work of J. S. Bach from the output of a Markov chain.

As previously noted, figure 1c exhibits, when the conditional entropy is employed as the test statistic, a sharp transition from a rate of rejection of $100 \%$ for hypotheses of Markov order one or less to a rate of rejection of $10 \%$ or lower for hypotheses of Markov order of two or more. Figure $1 \mathrm{~b}$ shows for the same test statistic a far more gradual 


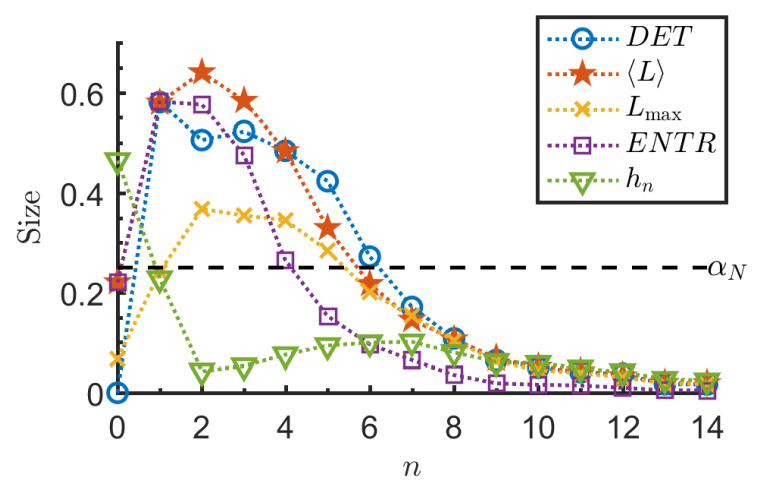

(a)

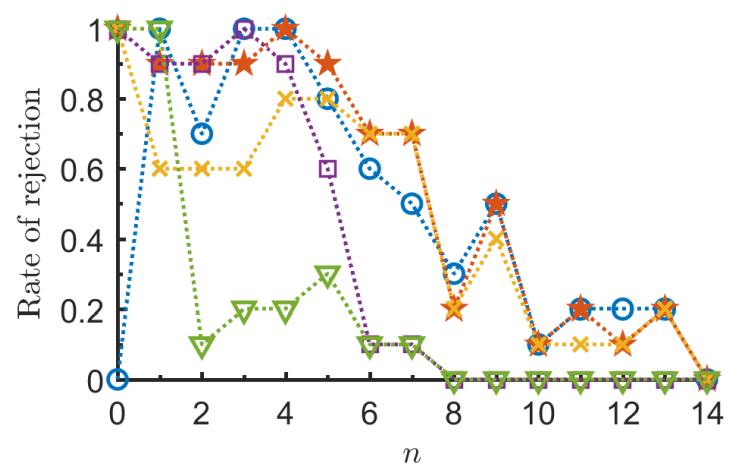

(b)

FIG. 3. Rates of rejection, at a nominal size $\alpha_{N}=0.25$, of the null hypothesis that time series arose from a Markov chain of order $n$ for (a) realisations of 1000 Markov chains of order $n$; and (b) ten compositions by J. S. Bach.

565 transition from a perfect rate of rejection for hypotheses of Markov order one or less to a rate of rejection of $10 \%$ or lower for hypotheses of Markov order of six. The failure to reject, for original musical time series, hypotheses of Markov order two or more may suggest that Markov or570 der two sufficed to capture an important part of musical compositions; perhaps an aspect associated with simple music composition conventions. It would be interesting to see whether the pattern persists for shorter or longer musical time series, for pieces by other composers, and 575 for different styles.

The failure to reject, using any test statistic, hypotheses of order nine or greater is probably mostly due to the brevity of the time series and the large number of distinct symbols which they feature. (See table I for quantifica580 tion of these and other properties of the compositions.) This combination will tend to reduce the number of words of length $n$ which appear more than once in the time series, thus reducing the number of words which can possibly be observed to precede more than one distinct word 585 of length $n$. This reduces the number of states of the Markov chain at which there is any randomness, and so could make it harder to produce surrogates which differ significantly from the original data.
Figures 4 illustrate the reduction in randomness as 590 Markov order $n$ increased. The figures depict weighted, directed networks which represent the Markov chains constructed from Invention 1. The nodes of the networks representing a Markov chain of order $n$ are the words of length $n$ in the time series from which the Markov chain 595 is constructed. Nodes with outdegree $d^{+}$greater than or equal to two represent a state from which more than one transition is possible. Figure $4 \mathrm{a}$ represents the Markov chain of order $n=1$, and contains $\nu_{1}=30$ nodes, 19 of which have outdegree $d^{+}$greater than or equal to two. In 600 contrast, figure $4 \mathrm{~b}$, which represents the Markov chain of order $n=6$, contains $\nu_{6}=229$ accessible states, but only 6 have outdegree $d^{+}$greater than or equal to 2. Except at these 6 states the surrogate algorithm was constrained to follow deterministic chains without any element of ran605 domness. As order $n$ exceeded the maximum diagonal line length $L_{\max }$, no word of length $n$ occurred more than once, and all randomness disappeared. The network which represented the Markov chain of order $n$ became a loop, and the "random" walk became a deterministic ${ }_{610}$ cycle. For Invention $1, L_{\max }=7$, and determinism takes over from order $n=8$.

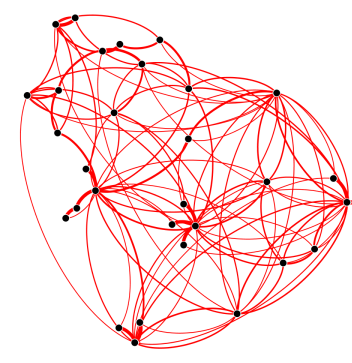

(a)

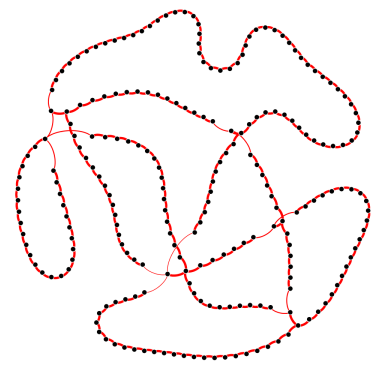

(b)
FIG. 4. Networks constructed from Invention 1 and depicted using the network visualisation software Gephi $i^{45}$. The nodes are the words of length: (a) $n=1$; and (b) $n=6$. The links are weighted according to the empirical probability of transitions from states, and the thickness with which they are drawn increases with weight. The links are directed and only traversed clockwise. Each surrogate for the hypothesis of Markov order $n$ comprised a random walk starting from the word of length $n$ which began the original composition.

States from which more than one transition was possible are also rare in Markov chains of order $n=6$ constructed from any of the other four inventions. Figure 5 shows the variation with $n$ of the fraction of nodes with outdegree $d^{+}$greater than or equal to two in networks representing Markov chains of order $n$ constructed from inventions by J. S. Bach. For the four inventions other than that from which figures 4 were constructed, 20 for $n=8$ the fraction of nodes with outdegree $d^{+}$greater than one was small but, although it is difficult to discern from the figure, nonzero. This implies, in particular, that the other inventions have maximum diagonal line length 


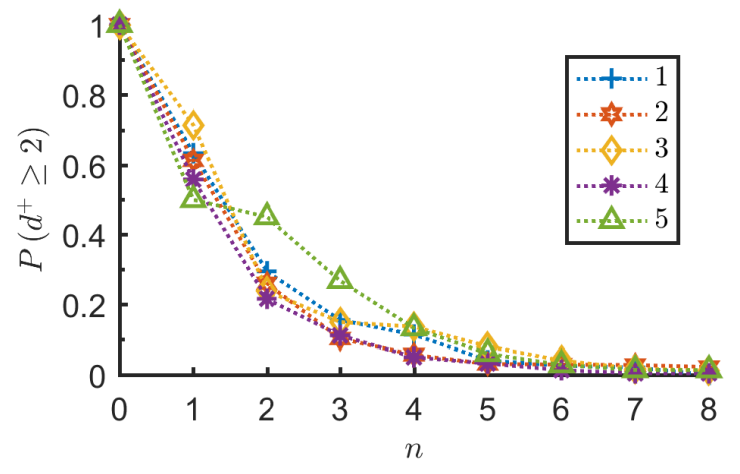

FIG. 5. The fraction of nodes with outdegree $d^{+}$greater than or equal to two in networks representing Markov chains of order $n$ constructed from each one of five inventions by J. S. Bach. For $n \geq 1$ this fraction, $P\left(d^{+} \geq 2\right)$, is equal to the fraction of words of length $n$ which immediately preceded, over the course of the Invention, more than a single word of length $n$.

$L_{\max }$ greater than seven.
TABLE I. Details of the compositions, and the time series to which they correspond, which we have considered. In this table, $N$ denotes the length of, and $\nu_{1}$ denotes the number of distinct values which appear in, the time series.

\begin{tabular}{cccccc}
\hline \hline \multicolumn{1}{c}{ Style } & Number & Catalogue & Key & $N$ & $\nu_{1}$ \\
\hline Invention & 1 & BWV 772 & C major & 242 & 30 \\
Invention & 2 & BWV 773 & C minor & 249 & 31 \\
Invention & 3 & BWV 774 & D major & 246 & 28 \\
Invention & 4 & BWV 775 & D minor & 255 & 43 \\
Invention & 5 & BWV 776 & Eb major & 228 & 20 \\
Sinfonia & 1 & BWV 787 & C major & 247 & 58 \\
Sinfonia & 2 & BWV 788 & C minor & 259 & 38 \\
Sinfonia & 3 & BWV 789 & D major & 238 & 55 \\
Sinfonia & 4 & BWV 790 & D minor & 226 & 46 \\
Sinfonia & 5 & BWV 791 & Eb major & 221 & 45 \\
\hline \hline
\end{tabular}

of surrogate data conditioned on their having, under the null hypothesis, a sufficient statistic coinciding with that 660 of the original data. This practice increases the computational burden of hypothesis testing but also improves accuracy and increases power ${ }^{19}$.

\section{ACKNOWLEDGMENTS}

MS is supported by the Australian Research Council We have investigated the null hypothesis that Bach's brain was actually a Markov chain. The RQA measure $L_{\max }$ allowed rejection of null hypotheses that compositions by J. S. Bach arose from Markov chains more ${ }_{630}$ consistently than did the conditional entropy, an established test statistic for Markov order. The advantages of RQA measures were particularly evident when power was treated as a function of the nominal size $\alpha_{N}$. However, the RQA measure $L_{\max }$ was also advantageous when ${ }_{635}$ power was assessed as a function of an estimate of the true power $\alpha$. The power of RQA measures in testing ${ }^{670}$ hypotheses of the origins of music data may arise from the importance to music of recurrence.

The failure to reject the hypothesis of a Markov chain 640 of Markov order greater than or equal to two when using conditional entropy as a test statistic suggests that Markov chains of order two may capture an important aspect of composition conventions. Analogous patterns for Markov order nine or greater when using the RQA

${ }_{645}$ measure $L_{\max }$ spring from the shortness of, and the large number of distinct symbols featured in, the time series constructed from the ten compositions which we have considered.

Van der Heyden et al. ${ }^{19}$ also used the method of sur${ }_{650}$ rogates to test Markov order. Their work, which used the conditional entropy as a test statistic, inspired and guided our contribution. Although our comparison of conditional entropy and different RQA measures is, in it- 68 self, interesting and useful, a valuable next step would be 655 to follow Van der Heyden et al. ${ }^{19}$ further in using constrained, rather than unconstrained, surrogate methods. Constrained surrogate methods involve generating sets through Discovery Project DP140100203. JMM was supported by the UWA Prescott, the CSIRO OCE and the CSIRO-UWA Complex Engineering Systems PhD scholarships.

\section{Appendix A: Ten compositions by J. S. Bach}

This appendix provides, via table I, details of the ten compositions which we considered. For $j \geq 1$ let $\nu_{j}$ denote the number of words of length $n$ in the time series once it has been appended as described in section III B, and let $\nu_{0}=\nu_{1}$. With these choices, for each $j \geq 0$, the 5 number of accessible states in the Markov chain of order $j$ built from the time series is equal to $\nu_{j}$. Figures 6 show that as the order $n$ increases the number $\nu_{n}$ of accessible states in the Markov chain of order $n$ approaches from below the length $N$ of the time series from which it was constructed. When the order $n$ exceeds the maximum diagonal line length $L_{\max }, \nu_{n}=N$ and the Markov chain of order $n$ comprises a deterministic loop. Figure 6a illustrates this for Inventions, while figure $6 \mathrm{~b}$ illustrates this for Sinfonias.

\section{REFERENCES}

\footnotetext{
${ }^{1}$ S. J. Mithen, The Singing Neanderthals: The Origins of Music, Language, Mind, and Body. Harvard University Press, 2005.
} 


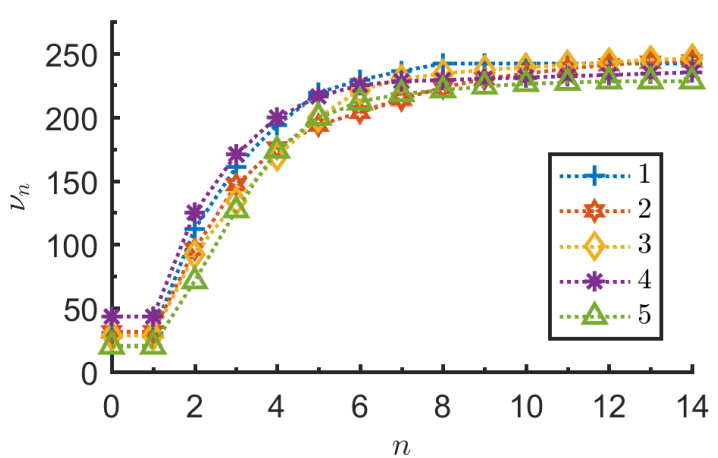

(a)

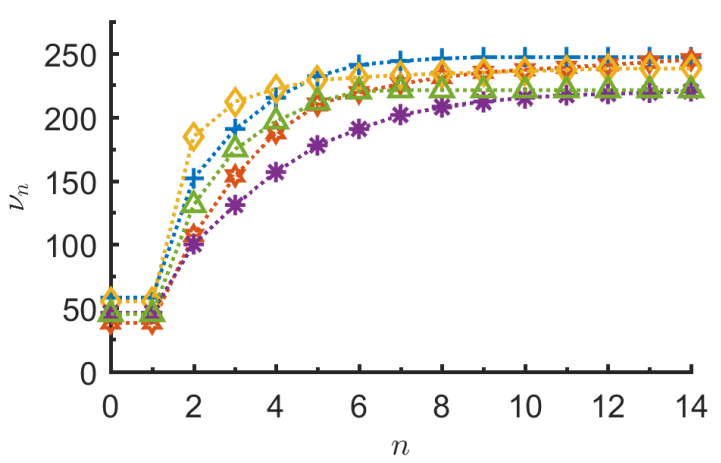

(b)

FIG. 6. Number $\nu_{n}$ of distinct words of length $n$ in time series of five: (a) Inventions; and (b) Sinfonias; by J. S. Bach. The compositions have been appended as described in section III B, and $\nu_{0}$ is defined as $\nu_{0} \triangleq \nu_{1}$.

${ }^{2} \mathrm{M}$. Trimble and D. Hesdorffer, "Music and the brain: The neuroscience of music and musical appreciation," BJPsych interna690

tional, vol. 14, no. 2, p. 28, 2017.

${ }^{3}$ T. Schäfer, P. Sedlmeier, C. Städtler, and D. Huron, "The psychological functions of music listening," Frontiers in psychology, vol. 4, 2013.

${ }^{4}$ X. F. Liu, K. T. Chi, and M. Small, "Complex network structure

musical compositions: Algorithmic generation of appealing music," Physica A: Statistical Mechanics and its Applications, vol. 389 , no. 1 , pp. 126-132, 2010.

${ }^{5}$ A. Klapuri and M. Davy, Signal processing methods for music transcription. Springer Science \& Business Media, 2007.

${ }^{6}$ J. Theiler and D. Prichard, "Using "surrogate surrogate data" to calibrate the actual rate of false positives in tests for nonlinearity in time series," in Nonlinear dynamics and time series: Building a bridge between the natural and statistical sciences, ser. Fields Institute Communications, 11., C. D. Cutler and D. T. Kaplan,

705 Eds. Providence, R.I.: American Mathematical Society, 1997, pp. 99-112.

${ }^{7}$ G. Schwarz et al., "Estimating the dimension of a model," The annals of statistics, vol. 6, no. 2, pp. 461-464, 1978.

${ }^{8}$ S. Sinha, M. D. Schroeder, U. Unnerstall, U. Gaul, and E. D. Siggia, "Cross-species comparison significantly improves genomewide prediction of cis-regulatory modules in drosophila," $B M C$ bioinformatics, vol. 5, no. 1, p. 129, 2004.

${ }^{9}$ A. Krogh, M. Brown, I. S. Mian, K. Sjölander, and D. Haussler, "Hidden markov models in computational biology: Applications to protein modeling," Journal of molecular biology, vol. 235 , no. 5, pp. 1501-1531, 1994.
${ }^{10}$ L. Saul and F. Pereira, "Aggregate and mixed-order markov models for statistical language processing," arXiv preprint cmplg/9706007, 1997.

${ }^{720}{ }^{11}$ P. Singer, D. Helic, B. Taraghi, and M. Strohmaier, "Detecting memory and structure in human navigation patterns using markov chain models of varying order," PloS one, vol. 9, no. 7, p. e102070, 2014.

12 J. Sakellariou, F. Tria, V. Loreto, and F. Pachet, "Maximum

725 entropy models capture melodic styles," Scientific Reports, vol. 7 , no. 1, p. 9172, 2017.

${ }^{13}$ A. Van Der Merwe and W. Schulze, "Music generation with markov models," IEEE MultiMedia, vol. 18 , no. 3 , pp. $78-85$, 2011.

${ }^{730}{ }^{14} \mathrm{~F}$. Pachet, "The continuator: Musical interaction with style," Journal of New Music Research, vol. 32, no. 3, pp. 333-341, 2003.

${ }^{15}$ D. Conklin, "Music generation from statistical models," in Proceedings of the AISB 2003 Symposium on Artificial Intelligence

735 and Creativity in the Arts and Sciences. Citeseer, 2003, pp. 30-35.

${ }^{16} \mathrm{M}$. Papapetrou and D. Kugiumtzis, "Markov chain order estimation with parametric significance tests of conditional mutual information," Simulation Modelling Practice and Theory, vol. 61, 740 pp. $1-13,2016$.

${ }^{17}$ P. Billingsley, "Statistical methods in Markov chains," The Annals of Mathematical Statistics, pp. 12-40, 1961.

${ }^{18}$ P. G. Hoel, "A test for Markoff chains," Biometrika, vol. 41, no. 3/4, pp. 430-433, 1954.

${ }_{745}{ }^{19}$ M. J. Van der Heyden, C. G. Diks, B. P. Hoekstra, and J. DeGoede, "Testing the order of discrete Markov chains using surrogate data," Physica D: Nonlinear Phenomena, vol. 117, no. 1-4, pp. 299-313, 1998.

${ }^{20}$ N. Marwan, N. Wessel, U. Meyerfeldt, A. Schirdewan, and

750 J. Kurths, "Recurrence-plot-based measures of complexity and their application to heart-rate-variability data," Physical review $E$, vol. 66, no. 2, p. $026702,2002$.

${ }^{21}$ M. Fukino, Y. Hirata, and K. Aihara, "Coarse-graining time series data: Recurrence plot of recurrence plots and its application

755 for music," Chaos: An Interdisciplinary Journal of Nonlinear Science, vol. 26, no. 2, p. 023116, 2016.

${ }^{22}$ J. Serra, X. Serra, and R. G. Andrzejak, "Cross recurrence quantification for cover song identification," New Journal of Physics, vol. 11, no. 9, p. 093017, 2009.

$760{ }^{23} \mathrm{M}$. Dirst and A. S. Weigend, "On completing J. S. Bach's last fugue," in Time Series Prediction: Forecasting the Future and Understanding the Past, A. S. Weigend and N. A. Gershenfeld, Eds. Addison-Wesley, 1993, pp. 151-177.

${ }^{24}$ T. O. Johnson, An analytical survey of the fifteen two-part inventions by J. S. Bach. University Press of America, 1982.

${ }^{25} \mathrm{~L}$. Dreyfus, Bach and the Patterns of Invention. Harvard University Press, 1996.

${ }^{26}$ D. B. Huron, Sweet anticipation: Music and the psychology of expectation. MIT Press, 2006.

$770{ }^{27}$ M. McCullough, K. Sakellariou, T. Stemler, and M. Small, "Regenerating time series from ordinal networks," Chaos: An Interdisciplinary Journal of Nonlinear Science, vol. 27, no. 3, p. 035814, 2017.

${ }^{28}$ E. N. Lorenz, "Deterministic nonperiodic flow," Journal of the atmospheric sciences, vol. 20, no. 2, pp. 130-141, 1963.

${ }^{29}$ A. S. Campanharo, M. I. Sirer, R. D. Malmgren, F. M. Ramos, and L. A. N. Amaral, "Duality between time series and networks," PloS one, vol. 6, no. 8, p. e23378, 2011.

${ }^{30}$ L. Hou, M. Small, and S. Lao, "Dynamical systems induced on networks constructed from time series," Entropy, vol. 17, no. 9, pp. 6433-6446, 2015.

${ }^{31}$ L. B. Meyer, Emotion and meaning in music. University of Chicago Press, 1956.

${ }^{32} \mathrm{C}$. E. Shannon and W. Weaver, The mathematical theory of communication. University of Illinois Press, 1964. 
${ }^{33}$ A. Papoulis and S. U. Pillai, Probability, random variables, and stochastic processes. Tata McGraw-Hill Education, 2002.

${ }^{34}$ N. Marwan, M. C. Romano, M. Thiel, and J. Kurths, "Recurrence plots for the analysis of complex systems," Physics reports, 790 vol. 438, no. 5, pp. 237-329, 2007.

${ }^{35}$ J.-P. Eckmann, S. O. Kamphorst, and D. Ruelle, "Recurrence plots of dynamical systems," EPL (Europhysics Letters), vol. 4, 8 no. 9 , p. $973,1987$.

${ }^{36}$ J. P. Zbilut and C. L. Webber, "Embeddings and delays as de795 vol. 171, no. 3-4, pp. 199-203, 1992.

${ }^{37}$ R. V. Donner, M. Small, J. F. Donges, N. Marwan, Y. Zou, 815 R. Xiang, and J. Kurths, "Recurrence-based time series analysis by means of complex network methods," International Journal

800 of Bifurcation and Chaos, vol. 21, no. 04, pp. 1019-1046, 2011.

${ }^{38}$ A. Krishnan, A. Giuliani, J. P. Zbilut, and M. Tomita, "Implications from a network-based topological analysis of ubiquitin 820 unfolding simulations," PLoS One, vol. 3, no. 5, p. e2149, 2008.
${ }^{39}$ M. Boyd, Bach. Oxford University Press, 2000.

$805{ }^{40}$ International Music Score Library Project (IMSLP): Petrucci Music Library, http://imslp.org, accessed Dec. 2017.

${ }^{41}$ T. Eerola and P. Toiviainen, "MIR in MATLAB: The MIDI toolbox," in Proceedings of the 5th International Conference on $\mathrm{Mu}$ sic Information Retrieval, 2004, pp. 22-27.

${ }^{42} \mathrm{M}$. Hewitt, Music theory for computer musicians. Nelson Education, 2008.

${ }^{43}$ B. Sericola, Markov chains: Theory and applications. John Wiley \& Sons, 2013.

${ }^{44} \mathrm{~J}$. Theiler and D. Prichard, "Constrained-realization MonteCarlo method for hypothesis testing," Physica D: Nonlinear Phenomena, vol. 94, no. 4, pp. 221-235, 1996.

${ }^{45}$ M. Bastian, S. Heymann, and M. Jacomy, "Gephi: An open source software for exploring and manipulating networks." in Proceedings of the Third International ICWSM Conference, 2009, pp. 361-362. 